\title{
NUMERICAL SIMULATIONS OF SPICULE ACCELERATION
}

\author{
N. Guerreiro, M. Carlsson, and V. Hansteen \\ Institute of Theoretical Astrophysics, University of Oslo, P.O. Box 1029 Blindern, N-0315 Oslo, Norway; \\ n.m.r.guerreiro@astro.uio.no, mats.carlsson@astro.uio.no,viggo.hansteen@astro.uio.no \\ Received 2012 October 17; accepted 2013 February 9; published 2013 March 15
}

\begin{abstract}
Observations in the $\mathrm{H} \alpha$ line of hydrogen and the $\mathrm{H}$ and $\mathrm{K}$ lines of singly ionized calcium on the solar limb reveal the existence of structures with jet-like behavior, usually designated as spicules. The driving mechanism for such structures remains poorly understood. Sterling et al. shed some light on the problem mimicking reconnection events in the chromosphere with a one-dimensional code by injecting energy with different spatial and temporal distributions and tracing the thermodynamic evolution of the upper chromospheric plasma. They found three different classes of jets resulting from these injections. We follow their approach but improve the physical description by including non-LTE cooling in strong spectral lines and non-equilibrium hydrogen ionization. Increased cooling and conversion of injected energy into hydrogen ionization energy instead of thermal energy both lead to weaker jets and smaller final extent of the spicules compared with Sterling et al. In our simulations we find different behavior depending on the timescale for hydrogen ionization/recombination. Radiation-driven ionization fronts also form.
\end{abstract}

Key words: Sun: chromosphere - Sun: transition region

\section{INTRODUCTION}

The solar chromosphere is filled with dynamic structures varying on various spatial and temporal scales. The structures are often classified according to their location and physical properties (Beckers 1968; Suematsu et al. 1995; Hansteen et al. 2006; Rouppe van der Voort et al. 2007). Some of the most prominent small-scale structures observable at the limb, designated as spicules, present a jet-like behavior. Spicules can be distinguished by their dynamics and timescales (De Pontieu et al. 2007b) and are subdivided into two types, I and II. These structures are observed in chromospheric spectral lines such as $\mathrm{H} \alpha$ and the $\mathrm{Ca}$ II $\mathrm{H}$ and $\mathrm{K}$ lines.

The two types of spicules differ mainly through their distinct dynamics (see Pereira et al. 2012): the type I spicules are characterized by timescales of the order of 150-400 s, velocities of $15-40 \mathrm{~km} \mathrm{~s}^{-1}$, and heights of 4-8 Mm, and an upward rise followed by a downward motion. Type II spicules, on the other hand, present shorter timescales (50-150 s), velocities of $30-110 \mathrm{~km} \mathrm{~s}^{-1}$, and reach greater heights; a particular characteristic of this category of spicules is their rapid fading in the chromospheric lines without presenting evidence of falling.

The broadly accepted driving mechanism for type I spicules is shock waves generated by collapsing granules or overshooting $p$-modes (De Pontieu et al. 2004; Hansteen et al. 2006; De Pontieu et al. 2007a; Heggland et al. 2007; Martínez-Sykora et al. 2009). While Matsumoto \& Shibata (2010) did not distinguish between type I and type II spicules, their Alfvén-wavedriven model aimed to reproduce features that have properties similar to type I spicules. Type II spicules are associated with violent events resulting most probably from reconnection. Martínez-Sykora et al. (2011) presented simulations where type II spicules evolve naturally from the dynamics of the model and are a result of a Lorenz force squeezing a flux tube.

Several numerical studies have been performed attempting to explain the driving mechanisms for the injection of chromospheric material into the corona (Suematsu et al. 1982; Shibata et al. 1982; Hollweg 1982; Shibata 1982; Sterling \& Mariska 1990; Sterling et al. 1991, 1993; for a good review, see also Sterling 2000).
Shibata et al. (1982) and Shibata (1982) studied the chromospheric response to energy depositions at different heights but in the absence of radiation losses and heat conduction. Sterling \& Mariska (1990) and Sterling et al. (1993) extended their work and included radiation losses and heat conduction and a more realistic treatment of the energy perturbation. They investigated the relationship between energy input and chromospheric ejections by varying the spatial and temporal distribution of energy deposition in the middle and upper chromosphere. They found as the result of the energy deposition three types of dynamic events, which they referred to as pressure-gradient jets, twocomponent jets, and gas plugs. They also found that this pure hydrodynamical mechanism can accelerate jets with chromospheric material that reach heights of $10-28 \mathrm{Mm}$.

In order to shed more light on the efficiency of the acceleration of spicules by a pressure gradient, we here revisit the Sterling et al. (1993) work, but employ a more realistic description of the non-LTE radiative transfer and population rate equations with a detailed treatment of hydrogen, calcium, and helium including non-equilibrium ionization and optically thick radiative losses.

The organization of this paper is as follows: a description of the method used by Sterling et al. (1993), the initial model, and our method is given in Section 2, the results are given in Section 3, and we finish with a discussion and conclusions in Section 4 .

\section{MODEL AND NUMERICAL METHOD}

Sterling et al. (1993) considered an initial atmosphere in hydrostatic equilibrium spanning over $20 \mathrm{Mm}$ from the photosphere to the corona including a rigid vertical magnetic flux tube with the lower end in the photosphere and the upper end open. The radius of the flux tube increased linearly from the photosphere up to about $2 \mathrm{Mm}$, where it was a factor of seven larger than in the photosphere, and the radius of the tube was kept roughly constant there above; see their Figure 1. The non-constant cross-sectional area was taken into account as an extra area factor in the one-dimensional (1D) equations of 
conservation of mass, momentum, and total energy,

$$
\begin{gathered}
\frac{\partial(A \rho)}{\partial t}+\frac{\partial(A \rho v)}{\partial z}=0, \\
\frac{\partial(\rho v A)}{\partial t}+\frac{\partial\left(\rho v^{2} A\right)}{\partial z}=-\rho g A-A \frac{\partial p}{\partial z}, \\
\frac{\partial E}{\partial t}+\frac{1}{A} \frac{\partial}{\partial z}\left[(E+p) v A-\kappa A \frac{\partial T}{\partial z}\right]=-\rho v g, \\
-L+S+E_{0} h(z, t),
\end{gathered}
$$

where $A$ is the (height dependent) area of the flux tube, $\rho$ density, $t$ time, $v$ velocity, $z$ height, $p$ gas pressure, $E$ total energy, $\kappa$ classical thermal conductivity, $L$ radiation losses, and $S$ the background heating. The gravitational acceleration is constant, $g=2.7 \times 10^{4} \mathrm{~cm} \mathrm{~s}^{-2}$. The term $E_{0} h(z, t)$ is a perturbative term to the total energy equation. The $E_{0}$ is the amplitude of the heating rate and $h(z, t)$ contains the spatial and temporal variations of the perturbative term; see Equation (8).

A perfect gas was considered with $\gamma=5 / 3$, and mean molecular weight, $\mu=1.3$, respecting the equation of state

$$
p=N k_{B} T,
$$

where $N$ is the particle number density and $k_{B}$ is the Boltzmann's constant. The constant mean molecular weight means that the ionization state of the plasma was assumed to be constant.

The radiation losses were treated differently depending on the temperature. The gas was assumed to be optically thin for $T>4 \times 10^{4} \mathrm{~K}$ with the total volumetric radiative losses given by

$$
L=N_{e} N_{p} \phi(T),
$$

where $N_{e}$ and $N_{p}$ are the electron and proton densities, respectively, and $\phi(T)$ is the radiative loss function.

The radiative loss function for $T>10^{5} \mathrm{~K}$ was originally given by Rosner et al. (1978) and later modified by Raymond (see Mariska et al. 1982).

The radiative loss function for $4.0 \times 10^{4}<T<5.0 \times 10^{5} \mathrm{~K}$ was given by (cgs units)

$$
\phi(T)=6.46 \times 10^{-35} T^{3} .
$$

The losses for $T<4.0 \times 10^{4} \mathrm{~K}$ were not treated solving the equations of radiative transfer; instead the chromospheric radiation loss rate was assumed to be given by (cgs units)

$$
L=4.9 \times 10^{9} \rho(z, t) .
$$

This expression was used in regions of positive pressure fluctuations. In regions of negative pressure fluctuations, no extra cooling or heating was assumed, only the constant background heating needed to keep the initial atmosphere constant in time in the absence of an explicit energy deposition; see below.

A computational grid of 400 fixed cells was employed, with the spacing ranging from 1 cell per $15 \mathrm{~km}$ in the photosphere to 1 cell per $10 \mathrm{~km}$ in the transition region. The grid becomes sparser in the corona where 1 cell per $280 \mathrm{~km}$ was considered. Due to the fixed nature of the grid, the steep gradients in the transition region were not fully resolved.

Open boundaries were used allowing the passage of material and waves into and out of the system and an absorbing layer was added in the first and last 10 grid cells to ensure that residual reflections at the boundaries did not enter into the main computational domain. The top boundary at $z=20 \mathrm{Mm}$ was kept at $7.7 \times 10^{5} \mathrm{~K}$.

The photosphere and chromosphere were assumed to be isothermal at $T=6230 \mathrm{~K}$. The initial temperature structure was kept constant in time in the absence of energy deposition by balancing radiation losses and heat conduction with a background heating in the initial model.

The injection of energy into the system was done by adding a perturbative term, $E_{0} h(z, t)$, to the energy equation, which represents the energy deposition in the chromosphere, varying in space and time and taking the form

$$
E_{0} h(z, t)=E_{0} \exp \left[-\left(\frac{z-z_{0}}{w}\right)^{2}\right] \sin \left(\frac{\pi t}{\tau}\right) .
$$

The coordinate $z_{0}$ is the deposition height, $t$ is the time coordinate, $\tau$ is one-half of one sine cycle of period $2 \tau$, and $w$ is the width parameter for the energy deposition. The heating term is switched on at $t=0$ and set to zero after $t=\tau$. By integrating the perturbative term over space and time, one finds the total energy deposited to be of the order,

$$
E_{\mathrm{tot}} \approx 2 \pi^{-\frac{1}{2}} w \tau A_{0} E_{0},
$$

where $A_{0}$ is the area of the flux tube at the height of the energy deposition.

It is now possible to treat the radiative losses with a higher degree of realism than what Sterling et al. (1993) could afford. In order to understand how the results of previous modeling attempts are changed when including a detailed treatment of the radiation and non-equilibrium ionization we use the RADYN 1D radiation hydrodynamics code. A comprehensive description can be found in Carlsson \& Stein (2002). In short, the code solves the 1D equations of mass, energy, momentum, and charge conservation together with the non-LTE radiative transfer and population rate equations implicitly on an adaptive grid (Dorfi \& Drury 1987).

In contrast to Sterling et al. (1993) we solve the internal energy equation formulated on a moving grid:

$$
\begin{aligned}
\frac{\partial}{\partial t}(\rho e) & +\frac{\partial}{\partial z}\left(\rho e\left(v-v_{\text {grid }}\right)\right)=-p \frac{\partial v}{\partial z}+\epsilon \frac{\partial v}{\partial z} \\
& -\frac{\partial}{\partial z}\left(F_{\mathrm{c}}+F_{\mathrm{r}}^{\mathrm{d}}+F_{\mathrm{r}}^{\mathrm{t}}+F_{\mathrm{h}}\right)+E_{0} h(z, t),
\end{aligned}
$$

where $\rho$ is the mass density, $e$ is the internal energy, $v$ is the bulk velocity, $v_{\text {grid }}$ is the grid velocity, $p$ is the gas pressure (radiation pressure is neglected), $\epsilon$ is the viscosity coefficient, $F_{\mathrm{c}}$ is the conductive flux, $F_{\mathrm{r}}^{\mathrm{d}}$ is the detailed radiative flux, $F_{\mathrm{r}}^{\mathrm{t}}$ is the thin radiative flux, $F_{\mathrm{h}}$ is the additional heating required to give the specific stationary temperature distribution, and $E_{0} h(z, t)$ is the energy deposition term. We assume a constant area flux tube and therefore we do not have an area factor in the equations of conservation of mass, momentum, and energy, in contrast to Sterling et al. (1993).

The detailed radiative flux, $F_{\mathrm{r}}^{\mathrm{d}}$, is the radiative flux integrated over wavelength calculated from solving the radiative transfer equation at 1487 frequency points covering continua from $4 \mathrm{~nm}$ to $4000 \mathrm{~nm}$ and spectral lines from hydrogen, helium, and calcium. For these elements we solve the rate equations (one per energy level considered) simultaneously with the equations of 


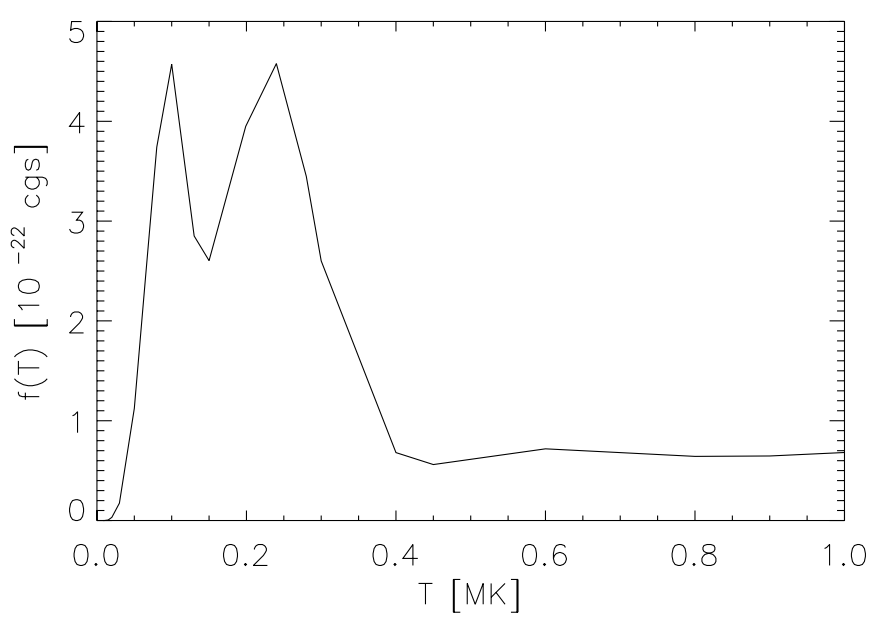

Figure 1. Optically thin loss function.

conservation of mass, momentum, energy, charge, and the grid equation. For each element, one of the rate equations is replaced by the particle conservation equation. Background opacity from other elements are treated in LTE. Hydrogen and singly ionized calcium are modeled by six-level atoms and helium with a ninelevel atom. At each grid-point we thus solve $6+6+9+5=26$ equations. The charge conservation equation, the rate equations and the particle conservation equations are given as follows:

$$
\begin{gathered}
\sum_{k} n_{i k} I_{i k}+n_{\mathrm{H}} n_{e}^{\mathrm{met}}\left(T, n_{e}\right)-n_{e}=0 \\
\frac{\partial n_{i k}}{\partial t}+\frac{\partial}{\partial z}\left(n_{i k}\left(v-v_{\text {grid }}\right)\right)=\sum_{j \neq i}\left(n_{j k} P_{j i k}-n_{i k} P_{i j k}\right), \\
\sum_{i} n_{i k}=A_{k} n_{\mathrm{H}},
\end{gathered}
$$

where $n_{i k}$ is the population density of energy level $i$ of element $k, I_{i k}$ is the ionization degree of energy level $i$ of element $k$ ( 0 for neutral, 1 for single ionized, etc.), $n_{\mathrm{H}}$ is the population density of hydrogen nuclei, $n_{e}^{\text {met }}\left(T, n_{e}\right)$ is the number of electrons per hydrogen nucleus as a function of temperature and electron density from elements not solved for explicitly, $n_{e}$ is the electron density, $P_{i j k}$ is the probability of a transition from energy level $i$ to energy level $j$ in element $k$, and $A_{k}$ is the abundance of element $k$ on a scale where the abundance of hydrogen is 1 .

We consider an initial model where the atmosphere is in hydrostatic equilibrium spanning over $10 \mathrm{Mm}$ from the photosphere to the corona where the top boundary is kept at $1 \mathrm{MK}$. In our scenario we do not include a rigid magnetic field with an area expansion factor. In order to convert our energy deposition per area to a total energy deposition, we use the values for $A_{0}$ from Sterling et al. (1993). In the comparison of our results it is important to keep in mind that we have a flux tube with constant area while their modeled flux tubes expand between the height of the energy deposition and the transition region and corona. This is discussed in Section 4.

Above a temperature of $2.0 \times 10^{4} \mathrm{~K}$ we include optically thin losses given by

$$
\frac{\partial F^{\mathrm{t}}}{\partial z}=L=n_{e} n_{\mathrm{H}} f(T),
$$

where $n_{e}$ and $n_{\mathrm{H}}$ are the number densities of electrons and hydrogen, respectively, $f(T)$ is a function of temperature and is

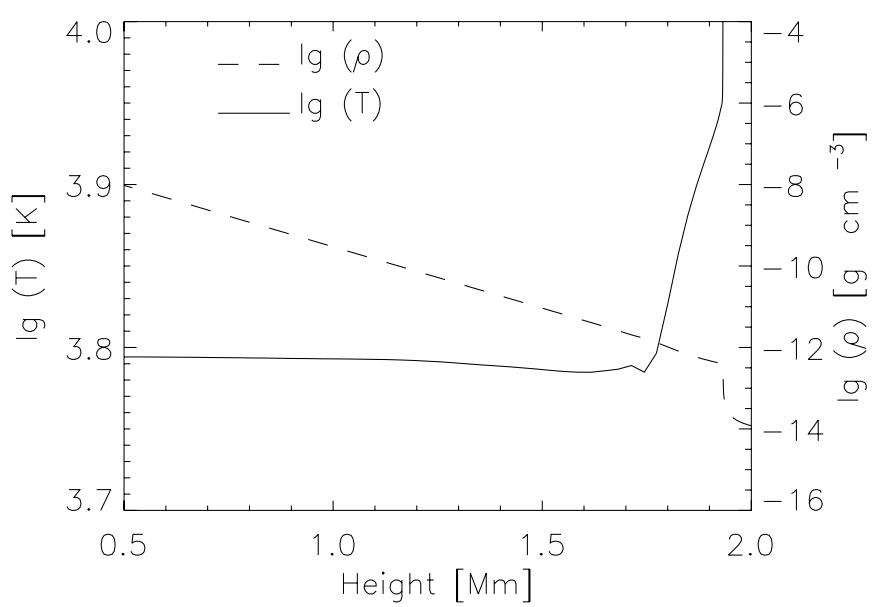

Figure 2. Temperature (solid) and density (dashed) in the initial chromospheric part of the atmospheric model.

computed using ionization and recombination rates given by Arnaud \& Rothenflug (1985) and Shull \& van Steenberg (1982) and collisional excitation rates given by the HAO-DIAPER atom data package (Judge \& Meisner 1994) including lines from $\mathrm{He}, \mathrm{C}, \mathrm{O}, \mathrm{Ne}$, and $\mathrm{Fe}$. The function $f(T)$ is pre-computed assuming ionization equilibrium; see Figure 1. Note that hydrogen radiative losses are calculated in detail and therefore not included in the optically thin radiative loss function. The chromospheric radiative losses are computed in detail for the most relevant elements in the chromosphere $(\mathrm{H}, \mathrm{He}$, and $\mathrm{Ca})$. The other elements are treated as background continua in LTE, using the Uppsala atmosphere program (Gustafsson 1973) but with updated photoionization cross-sections from the opacity project (TopBase 1995).

We use an adaptive grid with 191 points, allowing for a good resolution of the small structures that may form where steep gradients occur. In running the code, we find a maximum resolution of $121 \mathrm{~m}$ and a minimum resolution of $132 \mathrm{~km}$.

RADYN's upper boundary at $z=10 \mathrm{Mm}$ is transparent and the lower boundary condition at $138 \mathrm{~km}$ below $\tau_{500}=1$, is closed. The lower boundary condition reflects acoustic waves but the travel time between the boundary and the region of interest is more than $200 \mathrm{~s}$. A wave propagating downward from the height of the energy perturbation would be strongly damped by radiative losses in the photosphere and the much weakened reflected wave would not reach the region of interest before $t=400 \mathrm{~s}$. This implies that the reflections do not affect the results we will describe. We assume a model with an isothermal photosphere and chromosphere at about $T=6230 \mathrm{~K}$ as shown in Figure 2. In order for the starting solution to fulfill the energy equation, an extra term $\left(F_{\mathrm{h}}\right.$ in Equation (10)) is added that balances the detailed radiative losses. This extra heating term is kept constant in time per mass on a column mass scale. The transition region is initially at a height of slightly more than $1.9 \mathrm{Mm}$

We use the same method to inject energy into the chromosphere with the same spatial and temporal functions as Sterling et al. (1993); see Equation (8).

\section{RESULTS}

The injection of energy into the upper chromosphere and transition region gives rise to different features depending on the amount of energy injected, the location of injection and whether the energy injection is more or less localized. We started by using 

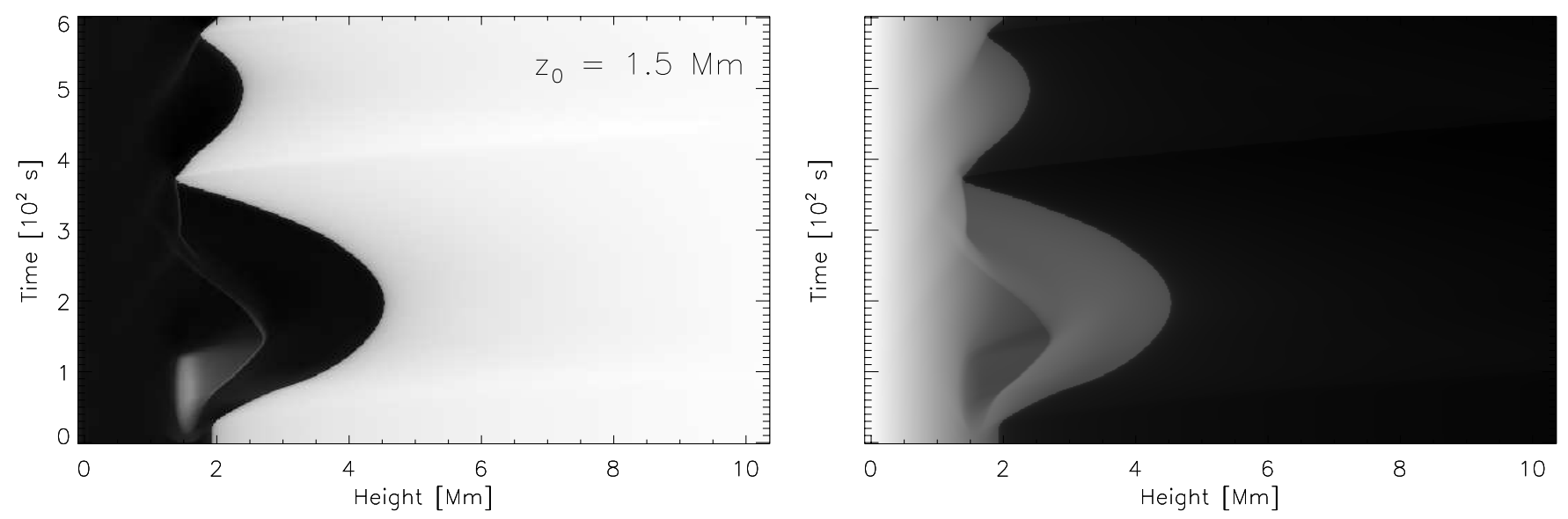

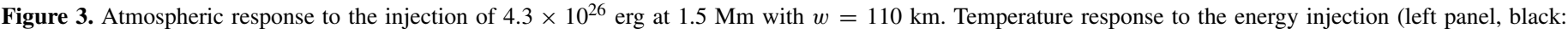
chromospheric temperature; white: coronal temperature). Density response to the energy injection (right panel, black: low density; white: high density).

the same values as Sterling et al. (1993). We injected energy varying from $3.0 \times 10^{25} \mathrm{erg}$ to $3.7 \times 10^{29} \mathrm{erg}$ at three different heights, $z_{0}=1.0 \mathrm{Mm}, z_{0}=1.5 \mathrm{Mm}$, and $z_{0}=1.75 \mathrm{Mm}$ with input durations of $\tau=120 \mathrm{~s}$ and $\tau=240 \mathrm{~s}$ and three different values for the width parameter, $w=110 \mathrm{~km}, w=220 \mathrm{~km}$, and $w=440 \mathrm{~km}$. We will first show one case and describe the temporal evolution in our simulation in some detail. We will then give the results for the whole grid of simulations and compare with the results of Sterling et al. (1993).

Figure 3 shows the result of the simulation with $E_{0}=$ $60 \mathrm{erg} \mathrm{cm}^{-3} \mathrm{~s}^{-1}$ (corresponding to a total energy of $4.3 \times$ $10^{26} \mathrm{erg}$ ) at $z_{0}=1.5 \mathrm{Mm}, \tau=120 \mathrm{~s}, w=110 \mathrm{~km}$, and $A_{0}=4.83 \times 10^{15} \mathrm{~cm}^{2}$. The injection of energy increases the temperature around the region of deposition but not to coronal values. At about $t=50 \mathrm{~s}$ after the beginning of the deposition, a shock forms in the deposition region and moves upward to a maximum height of about $2.7 \mathrm{Mm}$ leaving behind a tail of heated, lower density, material. The shock is weakened before it reaches the maximum height and it is pushed down by the material that is moving down after initially having been pushed up.

We can see two waves moving upward through the corona, one starting at about $t=50 \mathrm{~s}$ and the other at about $t=380 \mathrm{~s}$, when the transition region returns to the initial height and is propelled upward again. The waves are visible through the increase in temperature (left panel) and also through the effect on the density (right panel). The transition region reaches a maximum height of $4.5 \mathrm{Mm}$ at $t=220 \mathrm{~s}$, after that the transition region retracts to a position slightly lower than the initial position at about $t=380 \mathrm{~s}$ before it rebounds upward again, reaching a second maximum height of about $2.5 \mathrm{Mm}$, before it starts falling again.

At the time of maximum extent, $t=220 \mathrm{~s}$, the average density in the expanded region (the region between the original location of the transition region and the new location, 1.9-4.5 Mm) is $1.2 \times 10^{-12} \mathrm{~g} \mathrm{~cm}^{-3}$ and the average electron density is $2.1 \times 10^{11} \mathrm{~cm}^{-3}$.

Figure 4 shows the right-hand side of the internal energy equation (Equation (10); left panels), the change of the internal energy components (Equation (15); middle panels), and the hydrogen ionization fraction and temperature (right panels) versus height at three instants in time: $t=2 \mathrm{~s}$ (first row), $t=4 \mathrm{~s}$ (second row), and $t=10 \mathrm{~s}$ (third row). The change in internal energy is split into three components:

$$
\delta e=\delta e_{\mathrm{t}}+\delta e_{\mathrm{i}, \mathrm{H}}+\delta e_{\mathrm{i}, \mathrm{o}},
$$

where $\delta e$ is the total change of internal energy, $\delta e_{\mathrm{t}}$ is the change in thermal energy, $\delta e_{i, \mathrm{H}}$ is the change in hydrogen ionization and excitation energy, and $\delta e_{i, o}$ is the change in ionization and excitation energy of other elements than hydrogen.

Figure 4 shows the very early development after the start of the energy deposition into the atmosphere. The first row of panels for $t=2 \mathrm{~s}$ shows that the energy injected into the chromosphere at that point in time is not being radiated away (first panel from the left); instead it contributes mostly to increasing the thermal energy and, to a lesser degree, the hydrogen ionization and excitation (middle panel). The change in thermal energy means that the temperature increases (right panel) while the hydrogen ionization fraction remains unchanged because the ionization/recombination timescale at this height is several seconds. The left panel also shows a small positive heating from the detailed radiation. A closer analysis shows that this radiative heating takes place in the Balmer continuum which contributes to increasing the temperature, ionization, and excitation. The increase in temperature causes an increase in the collisional rate from the ground state of hydrogen to the first excited level and then there is absorption in the Balmer continuum. At about $1.95 \mathrm{Mm}$ we see the transition region, as yet not displaced.

At $t=4 \mathrm{~s}$ (second row) the change in the internal energy is clearly larger than the energy injected due to radiative heating (left panel). The energy injected is not being radiated away and the increase in the total internal energy is mainly in the form of an increase of the ionization and excitation energy and not an increase of the temperature (middle panel). The right panel shows that the increase of temperature is inhibited in the region where the ionization rate starts increasing.

At $t=10 \mathrm{~s}$ (bottom panels) the injected energy is partially balanced by the detailed radiation and thin radiation (left panel). The thin radiation starts becoming more efficient with the temperature increase. The energy not radiated away is spent ionizing and exciting hydrogen and, to a lesser degree, increasing the temperature. The right panel shows an increase in the ionization degree but not the temperature. The switch from radiative heating to radiative cooling, essentially in the Balmer continuum, visible in the left panels from $t=4 \mathrm{~s}$ to $t=10 \mathrm{~s}$ results from the increase in proton density because of the increased ionization. The increased proton density leads to increased recombination and therefore increased emission in the Balmer continuum. At this point in time the transition region has not been propelled upward and remains at the initial height. 

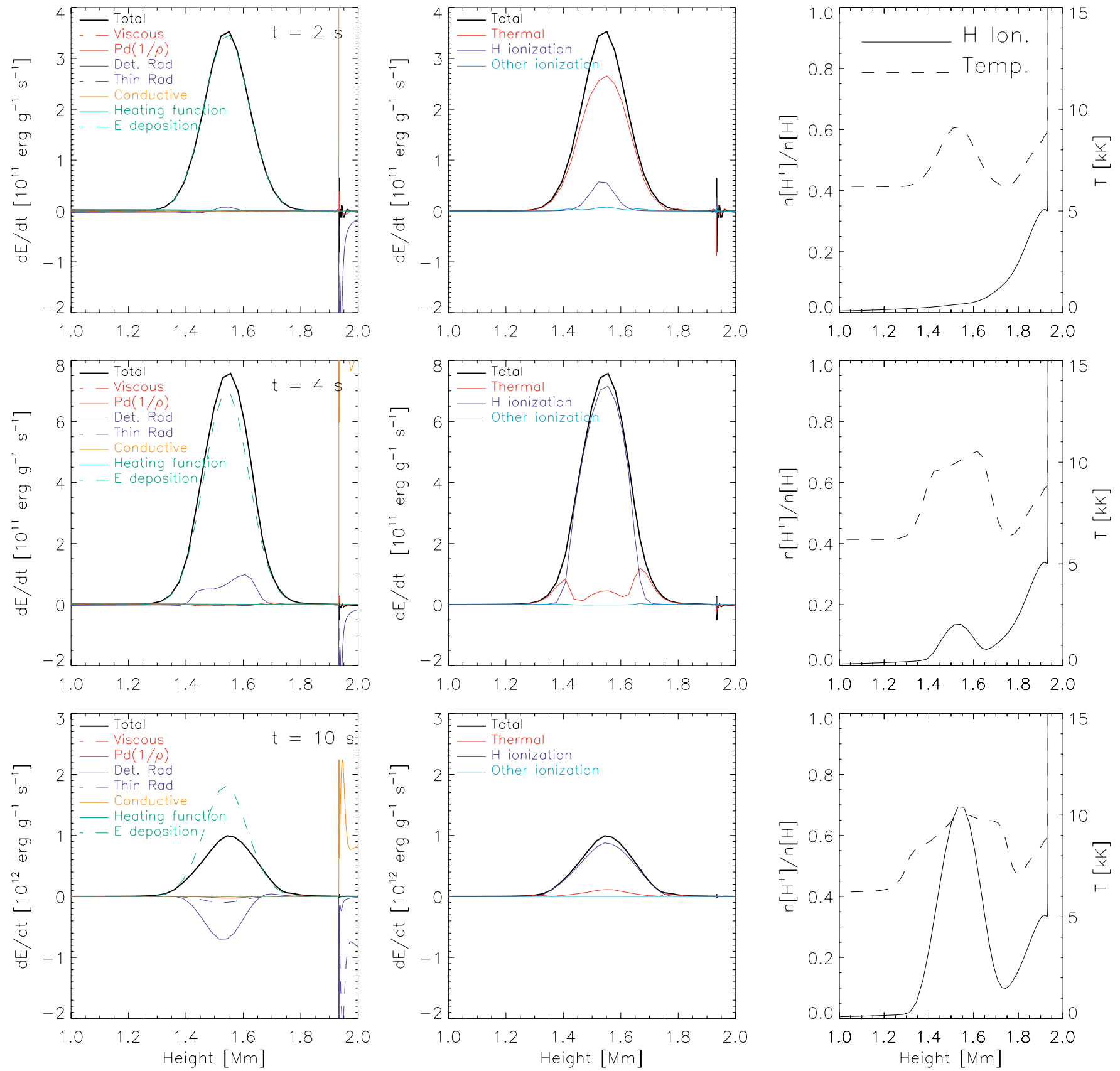

Figure 4. Energy balance when injecting $4.3 \times 10^{26} \mathrm{erg}$ at $z_{0}=1.5 \mathrm{Mm}$ at $t=2 \mathrm{~s}$ (top row), $t=4 \mathrm{~s}$ (middle row), and $t=10 \mathrm{~s}$ (bottom row). Time derivative of the internal energy and the heating terms against height (left panels). The heating terms are the right-hand side of Equation (10). Rate of change of the total internal energy (black), viscous heating (dashed red), compression work (red), detailed radiative heating (blue), thin radiative heating (dashed blue), conductive heating (yellow), heating function to keep the initial atmosphere stationary (green), and finally the energy deposition term (dashed green). Time derivative of the internal energy change (middle panels): Rate of change of total internal energy (black), thermal energy (red), hydrogen ionization and excitation energy (dark blue), and ionization and excitation energy for elements other than hydrogen (turquoise). Hydrogen ionization degree (right panels, solid) and temperature (dashed) vs. height.

Figure 5 shows the evolution a little later, at $t=14-22 \mathrm{~s}$. During this time interval an ionization front is formed at the upper edge of the heated region and moves up toward the transition region. Initially, at $t=14 \mathrm{~s}$ (top row), detailed radiation works as the main cooling mechanism, followed by thin radiation and by expansion work (top left panel). The cooling is not sufficient to radiate away all the energy dumped into the chromosphere. The main contributions to the detailed radiative cooling come from the Ly $\alpha$ line, the Lyman continuum, and the Balmer continuum. The energy not radiated away goes into ionizing hydrogen and increasing the thermal energy of the gas as shown in the middle panel. The input energy continues to ionize hydrogen until it is fully ionized at which point we get a rapid increase of the temperature. At $t=14 \mathrm{~s}$, hydrogen is almost fully ionized in the center of the deposition region and this is why most of the energy deposition goes into increasing the temperature there while on both sides we see mainly an increase in the hydrogen ionization.

At $t=18 \mathrm{~s}$ most of the energy injected into the chromosphere is balanced by detailed radiation, thin radiation, and expansion work. The cooling by thin radiation has increased in importance compared with earlier because of the increased temperature. 

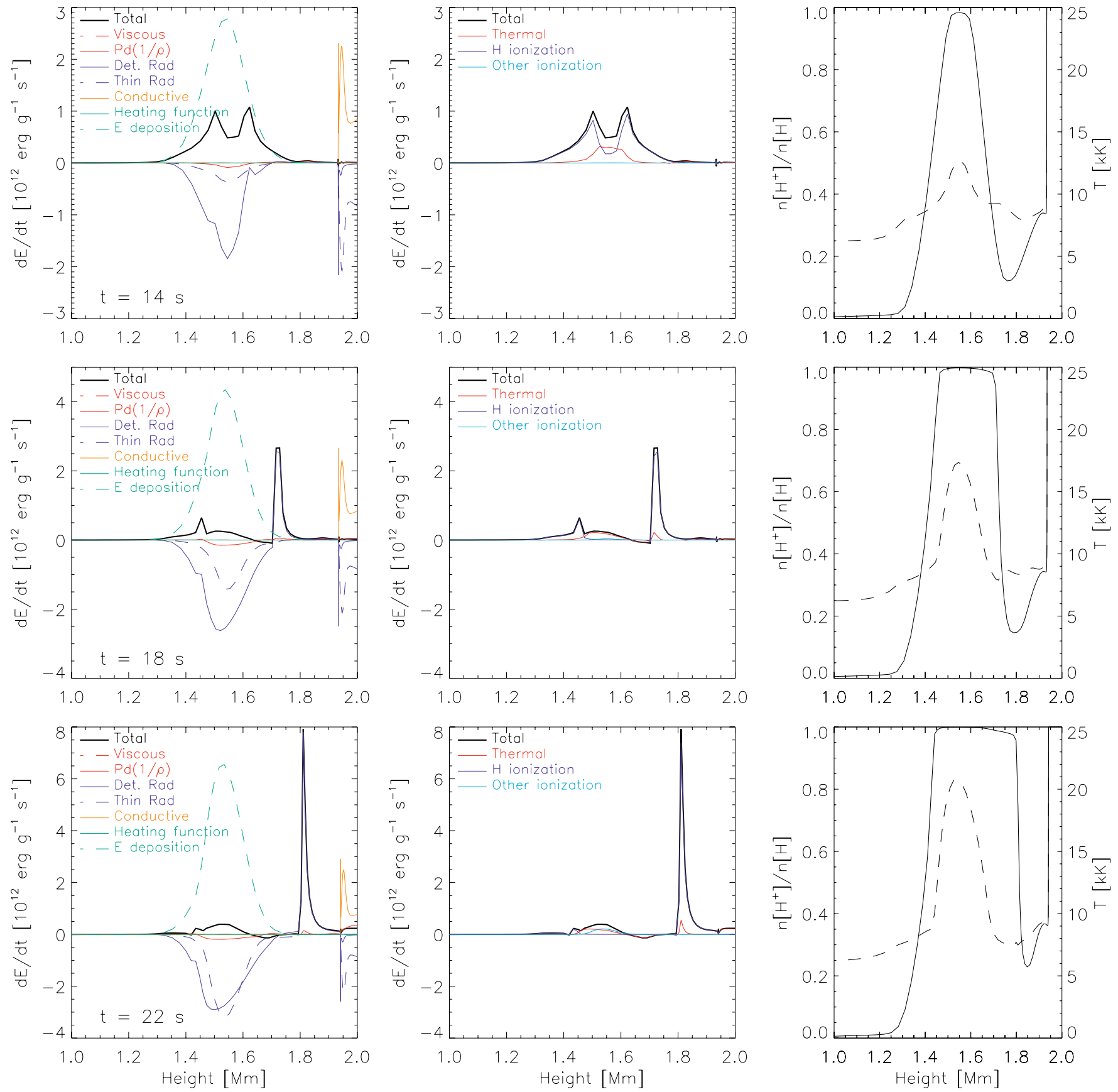

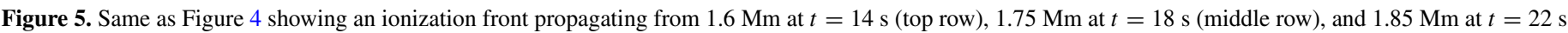
(bottom row).

The main contributions to the detailed radiation cooling are the Ly $\alpha$ line, the Lyman continuum, and the Balmer continuum. The Lyman radiation is absorbed at $1.7-1.75 \mathrm{Mm}$ height where there is still a significant fraction of neutral hydrogen. This radiative heating leads to an increase in the hydrogen ionization which makes the material transparent for the radiation that can progressively ionize the matter higher up. At $t=22 \mathrm{~s}$ (bottom panels) this ionization front is at $1.85 \mathrm{Mm}$.

Figure 6 shows the temperature, pressure, velocity and density evolution for the initial $78 \mathrm{~s}$. The deposition of energy around $z=1.5 \mathrm{Mm}$ increases the temperature (top left panel), resulting in an increase of the pressure gradient (top right panel). The increased pressure gradient exerts a force larger than gravity and matter is propelled upward. When the increased pressure gradient reaches the location of the transition region at $t=22 \mathrm{~s}$ (thick blue line), the transition region starts moving upward. The density decreases in the energy deposition region (bottom right panel), which leads to even more efficient heating; the energy deposition is specified per volume and the decreasing density thus leads to more energy deposited per particle. The upward velocity in the upper part of the energy deposition region increases with time and steepens into a shock at about $t=50 \mathrm{~s}$ (thick green line) giving rise to a temperature spike that propagates upward.

Figure 7 shows the temporal evolution for the time period $t=50-78 \mathrm{~s}$ showing the shock excited from the heating region propagating toward the moving transition region. For the temporal period shown in Figure 7, the energy deposition 

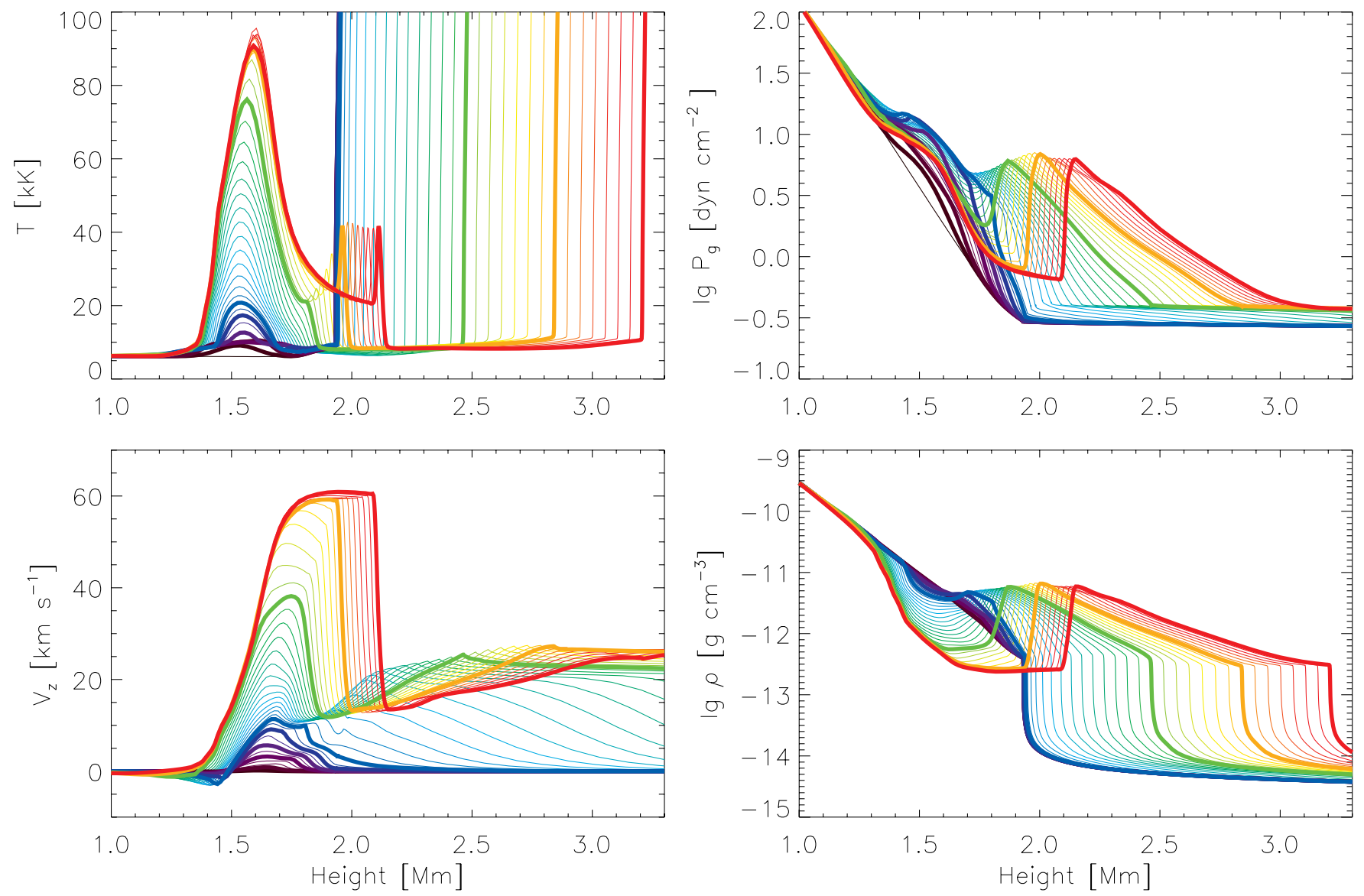

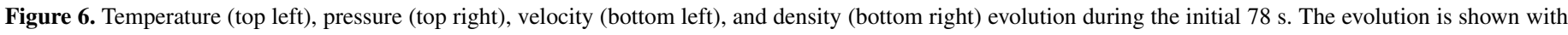

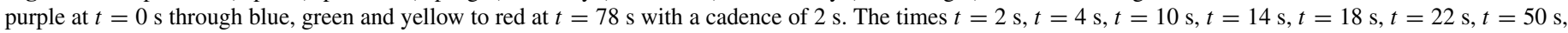
$t=64 \mathrm{~s}$, and $t=78 \mathrm{~s}$ displayed in Figures 4, 5, and 7 are highlighted with thicker lines.

is almost completely balanced by cooling-mainly in the form of optically thin radiative cooling but with a significant contribution from detailed radiation with contributions from the Ly $\alpha$ line, the Lyman continuum, and the Balmer continuum of hydrogen in the region between $1.35 \mathrm{Mm}$ and $1.46 \mathrm{Mm}$ and an even stronger contribution from the He II Ly $\alpha$ line in the region between $1.46 \mathrm{Mm}$ and $1.65 \mathrm{Mm}$. The middle column shows a more detailed view of the moving shock region showing how the viscous heating term increases in importance with the steepening of the shock. In the shock, the compression and viscous heating terms dominate over the cooling terms (middle panels) and the increase of internal energy goes into increased temperature (right panels) because hydrogen is all ionized already.

Figure 8 shows the temperature and density evolution resulting from the injection of energy at different heights, $z_{0}=$ $1.0 \mathrm{Mm}, 1.5 \mathrm{Mm}$, and $1.75 \mathrm{Mm}$. The width parameter is $w=440 \mathrm{~km}$ and the extension in time is $\tau=120 \mathrm{~s}$. The amplitude is the same, $E_{0}=2.083 \mathrm{erg} \mathrm{cm}^{-3} \mathrm{~s}^{-1}$, in all three cases, but since the area factor is assumed to increase with height in Sterling et al. (1993), the constant $E_{0}$ corresponds to different amounts of total energy deposited in their case; see Table 1. Note that the energy deposition is given per volume, which means that the energy deposition per particle peaks higher up in the atmosphere than $z_{0}$.

When the deposition is made at these heights a broad region of the chromosphere is heated, since the width parameter is large. The pressure increases and a pressure gradient between the heated region and the surrounding environment forms. The gradient propels material and the transition region upward.

With an energy deposition centered at $z_{0}=1.0 \mathrm{Mm}$ (top panels), the displacement of the transition region starts at about $20 \mathrm{~s}$; the maximum displacement happens at $t=134 \mathrm{~s}$ where the transition region is at $z=2.4 \mathrm{Mm}$. The transition region starts falling back and reaches its initial height at about $240 \mathrm{~s}$ and continues moving downward reaching heights slightly below the initial position. A rebound shock propels it upward again and it reaches the initial position again at about $t=480 \mathrm{~s}$.

With an energy deposition centered at $z_{0}=1.5 \mathrm{Mm}$ (middle panels), the dynamic evolution is rather similar but a bulge of rarefied heated material forms around the deposition region. The transition region is displaced to a maximum height of $4.0 \mathrm{Mm}$ at $t=157 \mathrm{~s}$ and it returns to its initial position after about $290 \mathrm{~s}$ for the first time. The heated bulge disappears toward the end of the energy deposition. When the transition region is bouncing back, shocks form in the chromosphere. The transition region relaxes to its initial position at about $600 \mathrm{~s}$.

The bottom panels show the case with an energy deposition centered at $z_{0}=1750 \mathrm{~km}$. Once again the energy deposition causes the displacement of mass and the transition region upward. For this particular case the heated bulge around the deposition region is easier to see than in the previous case and the gas is more rarified. The transition region is propelled to a maximum height of about $4.5 \mathrm{Mm}$ at $t=176 \mathrm{~s}$ and it returns to the initial position at about $t=310 \mathrm{~s}$; a rebound shock follows 

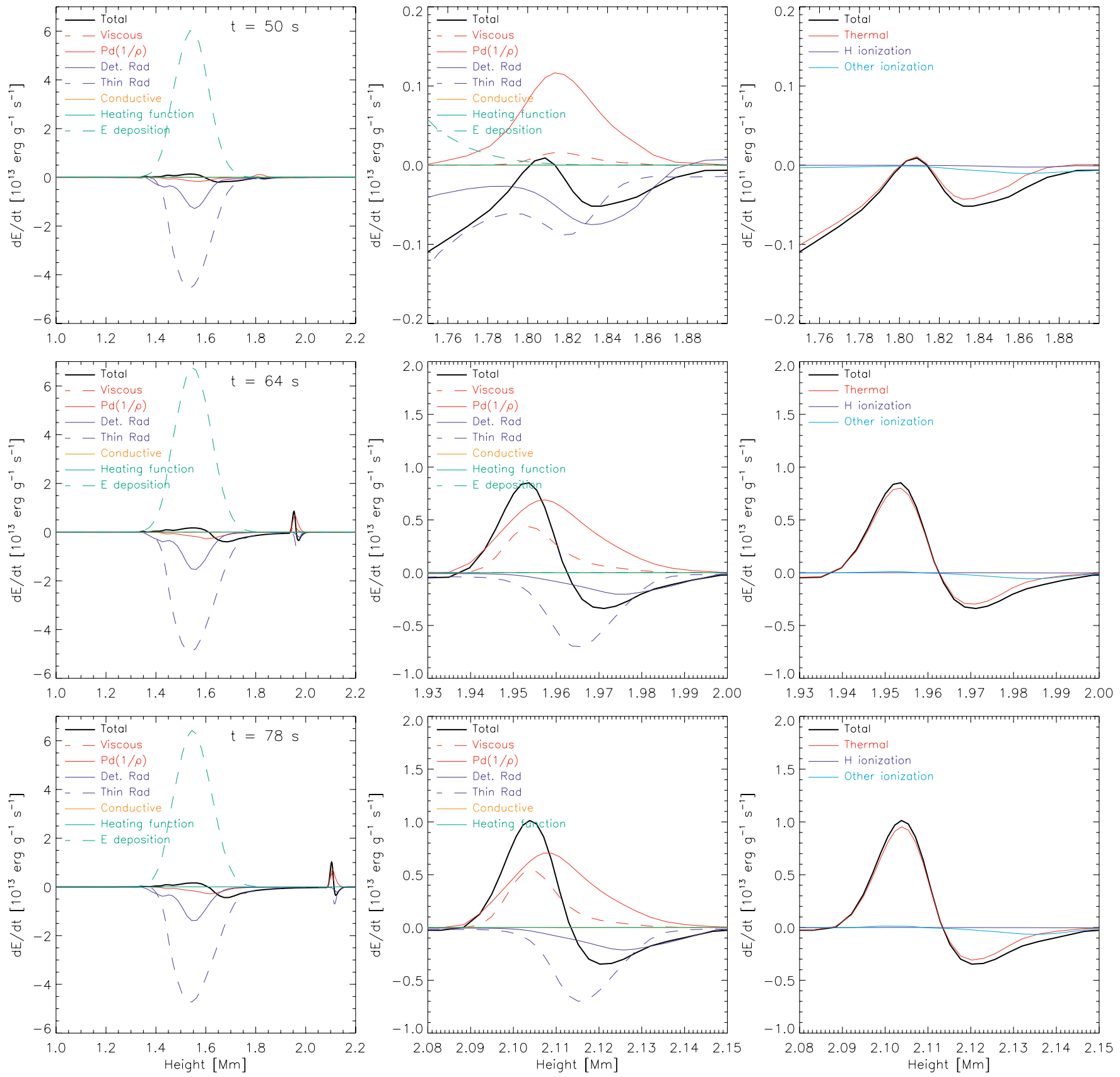

Figure 7. Energy balance in a shock excited slightly before $t=50 \mathrm{~s}$ in the heating region shown at $t=50 \mathrm{~s}$ (top row), $t=64 \mathrm{~s}$ (middle row), and $t=78 \mathrm{~s}$ (bottom row). Same as Figure 4 but excluding the hydrogen ionization degree and the temperature. The middle and right panels show an enlargement of the shock region. Note the different scales at $t=50 \mathrm{~s}$ compared with $t=64 \mathrm{~s}$ and $t=84 \mathrm{~s}$ in the middle and right panels.

that propels the transition region slightly upward again before it bounces back and relaxes toward the end of the run. A lower component of heated chromospheric gas forms in the deposition region before it cools off when the injection is switched off. As in the previous case small shocks form when the transition region is bouncing back the first time before it reaches the initial height. The density plot shows that the region where the heating is injected becomes rarified during the injection and it also shows that the density of the material displaced is kept roughly constant along its excursion.

Table 1 gives a summary of our experiments. In the left half of the table we give the input parameters and in the right half we give the resulting dynamical evolution deduced from a tracing of the position of the transition region: maximum height $(H)$, maximum velocity $\left(v_{\max }\right)$, the time for the maximum velocity $\left(t_{v_{\max }}\right)$, the minimum velocity or maximum downfall velocity $\left(v_{\min }\right)$, the maximum acceleration $\left(a_{\max }\right)$, and the maximum temperature in the energy deposition region $\left(T_{\text {chrom }}\right)$. The case described in detail above (Figures 4-7) is shown in bold; the cases shown in Figure 8 are shown in italics. We can also integrate the time derivative of the energy terms over the injection volume and time, $\tau$, and quantify where the total energy injected ends up. The results are shown in Table 2 with total radiative losses $(L)$, thin radiative losses $\left(L_{\text {thin }}\right)$, radiative losses calculated in detail $\left(L_{\mathrm{det}}\right)$ and $P d V$ work and the resulting change in internal energy $\left(E_{\text {Inter }}\right)$ and how that energy is distributed in changes in hydrogen ionization energy $\left(\mathrm{H}_{\mathrm{ion}}\right)$, helium ionization energy $\left(\mathrm{He}_{\mathrm{ion}}\right)$, ionization energy of 

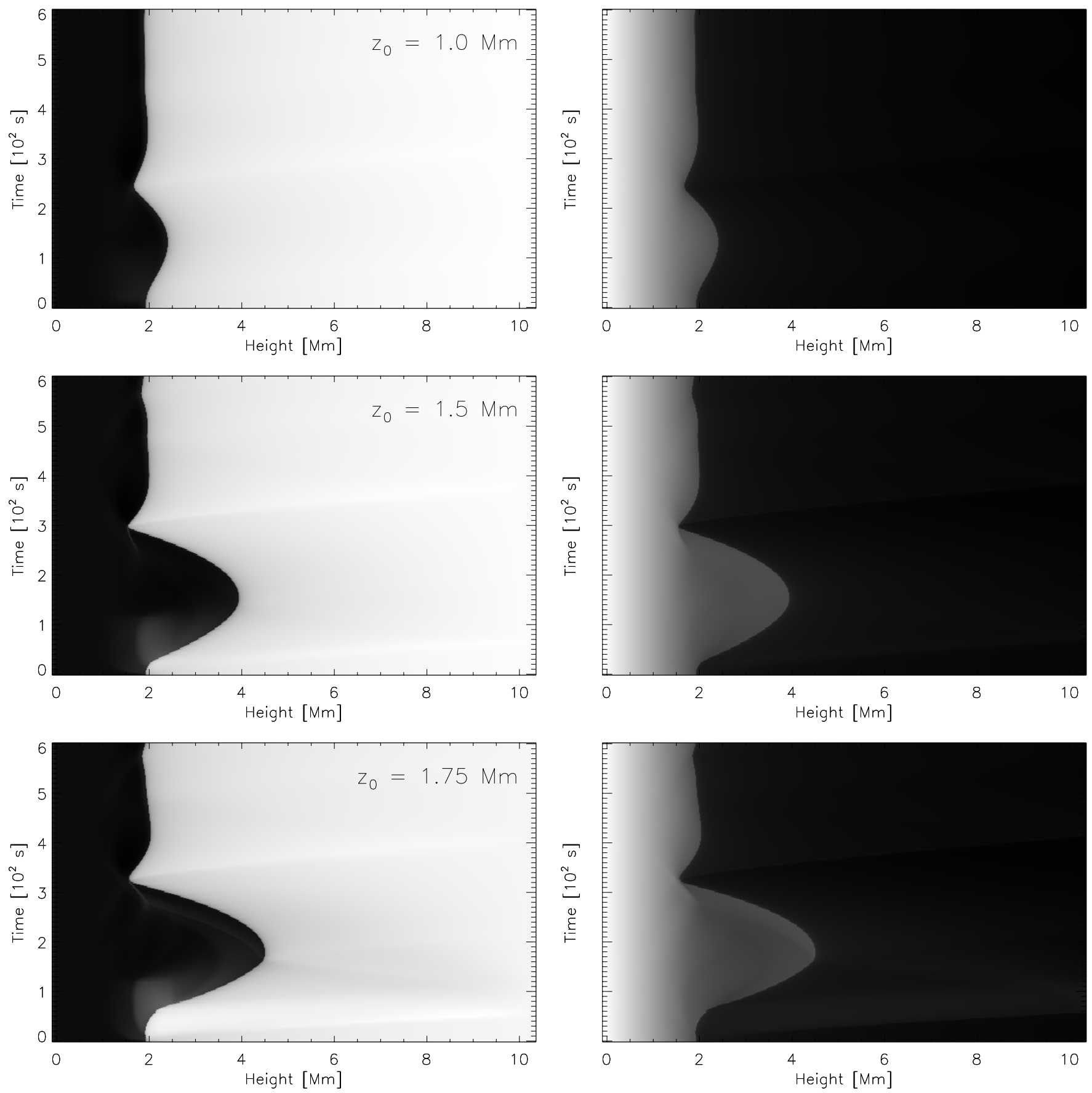

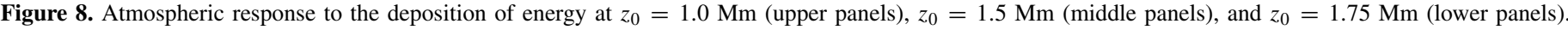

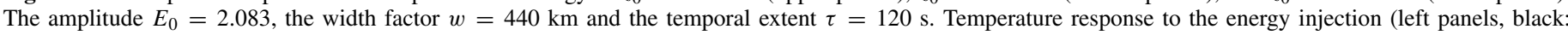
chromospheric temperature; white: coronal temperature). Density response to the energy injection (right panels, black: low density; white: high density)

other elements (Other $\left.{ }_{\text {ion }}\right)$, and thermal energy $\left(E_{\text {ther }}\right)$, all given in percentage of the total deposited energy .

The main difference between the three runs shown in Figure 8 (italics in Tables 1 and 2) is that the transition region is propelled to larger heights when the energy injection happens at a greater height. For the injection at $z_{0}=1.5 \mathrm{Mm}$ the maximum height is only $4.5 \mathrm{Mm}$, considerably less than the $10 \mathrm{Mm}$ for the corresponding case in Sterling et al. (1993; Column $H_{\mathrm{S}}$ in Table 1). In all three cases we get smaller velocities and a smaller maximum height than Sterling et al. (1993) found using their model. The main reason for this difference is that we get much lower temperature in the energy deposition region (Table 1,
Columns 12 and 13) and therefore a smaller pressure gradient. There are two main reasons for this difference: our more realistic description of the radiation gives larger radiative losses (both detailed losses and thin losses; see Section 4) and a large part of the injected energy is used to ionize hydrogen rather than increasing the thermal energy of the plasma; see Table 2.

We have made four experiments with an energy deposition centered at $z=1.0 \mathrm{Mm}$ with $\tau=120 \mathrm{~s}$ and $w=220 \mathrm{~km}$. They only differ in the amplitude of the energy deposition, $E_{0}$, with values ranging from 90 to $50000 \mathrm{erg} \mathrm{cm}^{-3} \mathrm{~s}^{-1}$. With increasing energy deposition we see increasing chromospheric temperature, acceleration, maximum velocity, and transition 
Table 1

Experiments

\begin{tabular}{|c|c|c|c|c|c|c|c|c|c|c|c|c|}
\hline $\begin{array}{l}z_{0}^{\mathrm{a}} \\
(\mathrm{Mm}) \\
(1)\end{array}$ & $\begin{array}{c}\text { Energy }^{b} \\
\text { (erg) } \\
\text { (2) }\end{array}$ & $\begin{array}{c}E_{0} \mathrm{c} \\
\left(\mathrm{erg} \mathrm{cm}^{-3} \mathrm{~s}^{-1}\right) \\
\text { (3) }\end{array}$ & $\begin{array}{l}\tau^{\mathrm{d}} \\
(\mathrm{s}) \\
(4)\end{array}$ & $\begin{array}{c}w^{\mathrm{e}} \\
(\mathrm{km}) \\
(5)\end{array}$ & $\begin{array}{c}H^{\mathrm{f}} \\
(\mathrm{Mm}) \\
(6)\end{array}$ & $\begin{array}{c}H_{\mathrm{S}}^{\mathrm{g}} \\
(\mathrm{Mm}) \\
(7)\end{array}$ & $\begin{array}{c}v_{\max }{ }^{\mathrm{h}} \\
\left(\mathrm{km} \mathrm{s}^{-1}\right) \\
(8)\end{array}$ & $\begin{array}{l}t_{v_{\max }} \\
(\mathrm{s}) \\
(9)\end{array}$ & $\begin{array}{c}v_{\min }{ }^{\mathrm{j}} \\
\left(\mathrm{km} \mathrm{s}^{-1}\right) \\
(10)\end{array}$ & $\begin{array}{c}a_{\max }{ }^{\mathrm{k}} \\
\left(\mathrm{km} \mathrm{s}^{-2}\right) \\
(11)\end{array}$ & $\begin{array}{c}T_{\text {chrom }}{ }^{1} \\
(\mathrm{kK}) \\
(12)\end{array}$ & $\begin{array}{l}T_{\mathrm{S}}^{\mathrm{m}} \\
(\mathrm{kK}) \\
(13)\end{array}$ \\
\hline \multirow[t]{5}{*}{1.0} & $3.0 \times 10^{25}$ & 2.083 & 120 & 440 & 2.4 & 3.0 & 6.2 & 59.9 & -10.9 & 0.2 & 7 & 15 \\
\hline & $6.7 \times 10^{26}$ & 90 & 120 & 220 & 2.6 & $\ldots$ & 7.9 & 62.9 & -17.6 & 0.7 & 8 & $\ldots$ \\
\hline & $1.5 \times 10^{28}$ & 2000 & 120 & 220 & 3.5 & $\ldots$ & 16.4 & 78.0 & -30.7 & 0.8 & 9 & $\ldots$ \\
\hline & $7.4 \times 10^{28}$ & 10000 & 120 & 220 & 6.2 & $\ldots$ & 35.7 & 65.7 & -46.2 & 1.7 & 19 & $\ldots$ \\
\hline & $3.7 \times 10^{29}$ & 50000 & 120 & 220 & 9.4 & $\cdots$ & 50.6 & 60.6 & -59.7 & 4.0 & 24 & $\cdots$ \\
\hline \multirow[t]{9}{*}{1.5} & $5.8 \times 10^{25}$ & 2.083 & 120 & 440 & 4.0 & 10 & 24.5 & 40.1 & -33.7 & 1.5 & 14 & 50 \\
\hline & $4.3 \times 10^{26}$ & 60 & 120 & 110 & 4.5 & $\ldots$ & 27.5 & 61.9 & -38.1 & 2.2 & 94 & $\ldots$ \\
\hline & $8.6 \times 10^{26}$ & 60 & 240 & 110 & 4.4 & $\ldots$ & 22.9 & 56.0 & -36.8 & 1.4 & 89 & $\ldots$ \\
\hline & $8.6 \times 10^{26}$ & 60 & 120 & 220 & 5.5 & $\ldots$ & 30.0 & 31.1 & -43.6 & 3.8 & 43 & $\ldots$ \\
\hline & $1.3 \times 10^{27}$ & 90 & 120 & 220 & 5.9 & $\ldots$ & 32.8 & 30.2 & -45.6 & 4.8 & 52 & $\ldots$ \\
\hline & $1.7 \times 10^{27}$ & 120 & 120 & 220 & 6.3 & $\ldots$ & 34.6 & 25.9 & -45.9 & 6.1 & 56 & $\ldots$ \\
\hline & $1.7 \times 10^{27}$ & 60 & 240 & 220 & 5.3 & $\ldots$ & 26.4 & 35.1 & -36.5 & 1.9 & 49 & $\ldots$ \\
\hline & $2.6 \times 10^{27}$ & 90 & 240 & 220 & 5.9 & $\ldots$ & 28.7 & 30.0 & -39.1 & 3.3 & 55 & $\ldots$ \\
\hline & $5.8 \times 10^{27}$ & 200 & 240 & 220 & 7.1 & $\ldots$ & 33.6 & 33.2 & -43.8 & 4.9 & 69 & $\ldots$ \\
\hline 1.75 & $7.2 \times 10^{25}$ & 2.083 & 120 & 440 & 4.5 & 12 & 41.7 & 70.2 & -41.2 & 3.5 & 29 & 63 \\
\hline
\end{tabular}

Notes.

a Deposition height.

b Total energy deposited.

c Amplitude of the heating rate.

${ }^{d}$ Length in time of energy deposition.

e Width parameter for the energy deposition.

${ }^{\mathrm{f}}$ Maximum height of the transition region.

g Maximum height of the transition region in Sterling et al. (1993).

${ }^{\mathrm{h}}$ Maximum velocity of the transition region.

i Time for the maximum velocity.

${ }^{\mathrm{j}}$ Maximum downfall velocity of the transition region.

$\mathrm{k}$ Maximum acceleration of the transition region.

${ }^{1}$ Maximum temperature in the energy deposition region.

$m$ Maximum temperature in the energy deposition region in Sterling et al. (1993).

Table 2

Energies

\begin{tabular}{|c|c|c|c|c|c|c|c|c|c|c|c|c|}
\hline $\begin{array}{l}z_{0} \\
(\mathrm{Mm}) \\
(1)\end{array}$ & $\begin{array}{c}E_{0} \\
\left(\operatorname{ergcm}^{-3} \mathrm{~s}^{-1}\right) \\
(2)\end{array}$ & $\begin{array}{l}\tau \\
(\mathrm{s}) \\
(3)\end{array}$ & $\begin{array}{c}w \\
(\mathrm{~km}) \\
(4)\end{array}$ & $\begin{array}{l}L^{\mathrm{a}} \\
\% \\
(5)\end{array}$ & $\begin{array}{c}L_{\text {thin }} \mathrm{b} \\
\% \\
(6)\end{array}$ & $\begin{array}{c}L_{\operatorname{det}}{ }^{c} \\
\% \\
(7)\end{array}$ & $\begin{array}{l}P d V^{\mathrm{d}} \\
\% \\
(8)\end{array}$ & $\begin{array}{c}E_{\text {Inter }} \mathrm{e} \\
\% \\
(9)\end{array}$ & $\begin{array}{c}\mathrm{H}_{\mathrm{ion}} \mathrm{f} \\
\% \\
(10)\end{array}$ & $\begin{array}{c}\mathrm{He}_{\text {ion }} \mathrm{g} \\
\% \\
(11)\end{array}$ & $\begin{array}{c}\text { Other }_{\text {ion }} \mathrm{h} \\
\% \\
(12)\end{array}$ & $\begin{array}{c}E_{\text {ther }}{ }^{\mathrm{i}} \\
\% \\
(13)\end{array}$ \\
\hline \multirow[t]{5}{*}{1.0} & 2.083 & 120 & 440 & 85.9 & 67.3 & 18.5 & 2.6 & 11.5 & 8.6 & 0 & 0 & 2.60 \\
\hline & 90 & 120 & 220 & 96.9 & 11.7 & 85.1 & 1.5 & 1.6 & 1.3 & 0 & 0 & 0.30 \\
\hline & 2000 & 120 & 220 & 99.3 & 6.80 & 92.5 & 0.3 & 0.4 & 0.3 & 0 & 0 & 0.03 \\
\hline & 10000 & 120 & 220 & 99.6 & 14.2 & 85.4 & 0.3 & 0.1 & 0.2 & 0 & 0 & -0.04 \\
\hline & 50000 & 120 & 220 & 99.8 & 43.9 & 56.0 & 0.4 & -0.3 & -0.2 & 0 & 0 & -0.05 \\
\hline \multirow[t]{9}{*}{1.5} & 2.083 & 120 & 440 & 79.9 & 41.1 & 38.9 & 3.2 & 16.9 & 14.9 & 0.1 & 0.2 & 1.9 \\
\hline & 60 & 120 & 110 & 92.2 & 64.3 & 28.0 & 2.9 & 4.9 & 3.1 & 0.5 & 0.6 & 1.2 \\
\hline & 60 & 240 & 110 & 92.5 & 66.6 & 25.9 & 3.2 & 4.3 & 2.5 & 0.5 & 0.6 & 1.2 \\
\hline & 60 & 120 & 220 & 96.4 & 55.7 & 39.2 & 1.6 & 3.6 & 2.6 & 0.3 & 0.3 & 0.7 \\
\hline & 90 & 120 & 220 & 96.0 & 62.8 & 33.2 & 1.3 & 2.7 & 1.9 & 0.2 & 0.3 & 0.5 \\
\hline & 120 & 120 & 220 & 96.7 & 67.0 & 29.7 & 1.1 & 2.2 & 1.5 & 0.2 & 0.2 & 0.4 \\
\hline & 60 & 240 & 220 & 94.8 & 58.7 & 36.4 & 1.8 & 3.1 & 2.2 & 0.3 & 0.3 & 0.6 \\
\hline & 90 & 240 & 220 & 96.1 & 65.2 & 30.9 & 1.5 & 2.4 & 1.6 & 0.2 & 0.2 & 0.5 \\
\hline & 200 & 240 & 220 & 97.6 & 73.9 & 23.7 & 1.0 & 1.4 & 0.9 & 0.2 & 0.2 & 0.3 \\
\hline 1.75 & 2.083 & 120 & 440 & 73.9 & 25.5 & 48.7 & 6.6 & 19.5 & 11.3 & 0.6 & 1.5 & 6.4 \\
\hline
\end{tabular}

Notes.

a Total radiative losses.

b Thin radiative losses.

c Radiative losses calculated in detail.

d $P d V$ work.

e Resulting change in internal energy.

${ }^{\mathrm{f}}$ Hydrogen ionization energy.

g Helium ionization energy.

h Ionization energy of other elements.

i Thermal energy. 
region excursion (Table 1) but also very large and increasing radiative losses-more than $99 \%$ of the injected energy is radiated away except for the lowest energy deposition case (Table 2).

We only have two experiments with energy deposition centered at $z=1.5 \mathrm{Mm}$ with the narrowest width: our reference case displayed in Figures 4-7 and in bold in the tables and a case with the energy deposition over twice the timespan. Both runs give a maximum temperature in the energy deposition region just under $100 \mathrm{kK}$. Our thin radiative loss function (see Figure 1) has a local maximum at about $100 \mathrm{kK}$. This means that if the temperature goes above $100 \mathrm{kK}$, the losses decrease and we get a very rapid temperature increase up to $150 \mathrm{kK}$. Even at higher temperatures we get a density decrease from the expansion leading to increased energy input per particle and the increase in the radiative loss function up to $250 \mathrm{kK}$ is not enough to prevent a runaway with temperatures rapidly reaching coronal values. This corresponds to the gas-plug cases of Sterling et al. (1991, 1993); a region with coronal temperatures in the region of energy deposition that slowly grows due to thermal conduction and a cool plug of material being pushed up between this region and the transition region. With the energy deposition concentrated in space $(w=110 \mathrm{~km})$ this happens already for $E_{0}=65 \mathrm{erg} \mathrm{cm}^{-3} \mathrm{~s}^{-1}$ in our simulations.

With the energy deposition more spread out $(w=220 \mathrm{~km})$ we have three experiments with $\tau=120 \mathrm{~s}$ and three experiments with $\tau=240 \mathrm{~s}$. The trend is the same as before with increasing maximum temperature in the deposition region, increasing acceleration, maximum velocity and transition region excursion with increasing energy deposition amplitude $E_{0}$. It can also be seen that doubling $E_{0}$ produces higher values than doubling the deposition time even though the two experiments have the same total energy deposition $\left(1.7 \times 10^{27}\right.$ erg assuming the crosssection area given in Sterling et al. (1993)).

The trends in the energy balance (Table 2) for the cases with the energy deposition at $z=1.5 \mathrm{Mm}$ are also similar to the cases with $z=1.0 \mathrm{Mm}$ : larger energy deposition leads to increased radiative losses. The balance shifts from radiative losses calculated in detail to thin radiative losses and increasingly so with increased energy deposition. This is correlated with the temperature reached in the energy deposition volume - the higher the temperature the more important are the thin radiative losses.

\section{DISCUSSION AND CONCLUSION}

Detailed analysis of our ensemble of runs allows us to come to some general conclusions. In the initial phase, the detailed energy balance depends on the density. When the energy injection is at $1500 \mathrm{~km}$ or above, the density is low, leading to long timescales for the ionization/recombination of hydrogen. The injected energy then leads to an immediate temperature increase followed by a slower temperature increase until all the hydrogen has been ionized. If the density is higher where the energy is injected, there is no such initial rapid temperature increase because the hydrogen ionization is not delayed.

After the ionization has started, an ionization front forms resulting from radiative heating in the Ly $\alpha$ and Lyman-continuum transitions. The radiation emitted around the deposition region is absorbed by the gas above. This process goes on until all the hydrogen between the energy deposition region and the transition region is ionized. The heating also creates disturbances which evolve into shocks. For injections at $1000 \mathrm{~km}$ and $1500 \mathrm{~km}$ the shocks formed never meet the transition region unless the deposition width is broad. Instead, they are pushed down by the material that is moving down after initially having been pushed up. The dumping of energy into the chromosphere propels matter and the transition region upward. Depending on the amount of energy injected into the atmosphere the gas responds differently but in general the injection of energy increases the pressure, forming a pressure gradient which propels the material and the transition region upward. Depending on the total energy, sometimes a bulge of heated material forms with temperatures above chromospheric and below coronal values.

Most of the energy deposited into the atmosphere is radiated away-for large energy deposition values this fraction is more than $99 \%$. When the energy deposition is at low heights, optically thick losses dominates while for higher deposition heights and larger energy deposition rates, optically thin losses are the most important ones. In all cases, the ionization energy of hydrogen plays an important role in limiting the amount of the remaining energy that goes into thermal energy.

Our results agree to some extent, at least qualitatively, with the results put forward by Sterling et al. (1993). Thus we find that the jets we mention above are what they refer to as pressure gradient jets. Sterling et al. (1993) also describe what they call two-component jets consisting of a lower, warm component and a cool, shock-generated component which catches up with the transition region. We have a hard time finding such twocomponent jets in our simulations. The third case described by Sterling et al. (1993), gas plug jets, we do find. When the energy deposition is high and the width is small we get a runaway situation: the temperature in the region of energy deposition reaches coronal values and we get a plug of cool material between the upward moving heated region and the transition region.

Despite a qualitative resemblance, the quantitative results are quite different. The excursions of the transition region are much smaller in our simulations than in theirs. For example, in the experiment where we inject $5.8 \times 10^{25}$ erg at $1.5 \mathrm{Mm}$ we get an excursion of the transition region of $4.0 \mathrm{Mm}$ while the same injection of energy in Sterling et al. (1993) shows a transition region excursion of $10 \mathrm{Mm}$. The smaller excursion comes from a lower maximum temperature in the energy deposition volume (14 kK compared with $50 \mathrm{kK}$ in the case mentioned) and therefore a smaller pressure gradient. There are two factors that restrict the temperature increase in our simulation: $80 \%$ of the injected energy is radiated away and of the $17 \%$ that goes into increasing the internal energy, only $1.9 \%$ ends up as increased thermal energy with the rest ending up as increased ionization energy, mostly of hydrogen. The latter effect is completely missing in the simulations of Sterling et al. (1993) since they assume a constant mean molecular weight and neglect ionization energy in the energy equation. It is more difficult to quantify the differences in the treatment of the radiative losses between our simulation and theirs. We treat in detail the optically thick radiation and also have a more realistic treatment of the optically thin radiation below $4.0 \times 10^{4} \mathrm{~K}$. We believe that both facts lead to higher radiative losses in our simulations than in theirs. One additional difference is that we do not include an expansion factor in our equations. However, it is hard to imagine that the inclusion of an expansion factor would make the excursion of the transition region larger since some of the energy must then go into horizontal expansion. We therefore conclude that it is hard to accelerate spicules to heights of the order of $10 \mathrm{Mm}$ by pure heating of the mid-chromosphere. 
This research was supported by the European Commission funded Research Training Network SOLAIRE. It was also supported by the Research Council of Norway through grants of computing time from the Norwegian Programme for Supercomputing. The research leading to these results has received funding from the European Research Council under the European Union's Seventh Framework Programme (FP7/2007-2013)/ ERC grant agreement No. 291058. We thank Bart De Pontieu for valuable discussions that have improved the paper.

\section{REFERENCES}

Arnaud, M., \& Rothenflug, R. 1985, A\&A, 60, 425

Beckers, J. M. 1968, SoPh, 3, 367

Carlsson, M., \& Stein, R. F. 2002, ApJ, 572, 626

De Pontieu, B., Erdélyi, R., \& James, S. P. 2004, Natur, 430, 536

De Pontieu, B., Hansteen, V. H., Rouppe van der Voort, L., van Noort, M., \& Carlsson, M. 2007a, ApJ, 655, 624

De Pontieu, B., McIntosh, S., Hansteen, V. H., et al. 2007b, PASJ, 59, 655

Dorfi, E. A., \& Drury, L. O. 1987, JCoPh, 69, 175

Gustafsson, B. 1973, UppAn, 5, 6

Hansteen, V. H., De Pontieu, B., Rouppe van der Voort, L., van Noort, M., \& Carlsson, M. 2006, ApJL, 647, L73

Heggland, L., De Pontieu, B., \& Hansteen, V. H. 2007, ApJ, 666, 1277
Hollweg, J. V. 1982, ApJ, 257, 345

Judge, P. G., \& Meisner, R. W. 1994, in ESA Special Publication 373, Solar Dynamic Phenomena and Solar Wind Consequences, The Third SOHO Workshop, ed. J. J. Hunt (Noordwijk: ESA), 67

Mariska, J. T., Doschek, G. A., Boris, J. P., Oran, E. S., \& Young, T. R., Jr. 1982, ApJ, 255, 783

Martínez-Sykora, J., Hansteen, V., DePontieu, B., \& Carlsson, M. 2009, ApJ, 701, 1569

Martínez-Sykora, J., Hansteen, V., \& Moreno-Insertis, F. 2011, ApJ, 736, 9

Matsumoto, T., \& Shibata, K. 2010, ApJ, 710, 1857

Pereira, T. M. D., De Pontieu, B., \& Carlsson, M. 2012, ApJ, 759, 18

Rosner, R., Tucker, W. H., \& Vaiana, G. S. 1978, ApJ, 220, 643

Rouppe van der Voort, L. H. M., De Pontieu, B., Hansteen, V. H., Carlsson, M., \& van Noort, M. 2007, ApJL, 660, L169

Shibata, K. 1982, SoPh, 81, 9

Shibata, K., Nishikawa, T., Kitai, R., \& Suematsu, Y. 1982, SoPh, 77, 121

Shull, J. M., \& van Steenberg, M. 1982, ApJ, 48, 95

Sterling, A. C. 2000 , SoPh, 196, 79

Sterling, A. C., \& Mariska, J. T. 1990, ApJ, 349, 647

Sterling, A. C., Mariska, J. T., Shibata, K., \& Suematsu, Y. 1991, ApJ, 381,313

Sterling, A. C., Shibata, K., \& Mariska, J. T. 1993, ApJ, 407, 778

Suematsu, Y., Shibata, K., Neshikawa, T., \& Kitai, R. 1982, SoPh, 75, 99

Suematsu, Y., Wang, H., \& Zirin, H. 1995, ApJ, 450, 411

TopBase 1995, The Opacity Project Team, The Opacity Project, Vol. 1 (Bristol: Institute of Physics Publishing) 